\title{
The Control of Search and Rescue Robots with the General Suppression Control Framework
}

\author{
Lau Henry Y. K. Ko Albert W. Y. \\ Department of Industrial and Manufacturing Systems Engineering, The University of Hong Kong, The Pokfulam of Hong Kong, \\ P. R. China \\ E-mail: hyklau@hku.hk
}

\begin{abstract}
The paper described the use of the general suppression control framework (GSCF) for the control and coordination of a team of search and rescue robots undertaking exploration operation. This study adopts the biological analogy of the human immune system to derive the GSCF having the behavior of immunological cells. The framework directs the coordination of these robots in tackling search and rescue operations in an unstructured environment. Simulation study is performed to demonstrate the effectiveness of the control framework.

Keywords: distributed control, modular robots, artificial immune system
\end{abstract}

\section{INTRODUCTION}

The interests to develop truly autonomous robots that have the ability to explore the environment and cooperate autonomously to achieve various tasks always prevail especially in search and rescue operations. These robots have the capabilities to navigate autonomously to search for specific targets and objects of interest, and to convey such finding to their neighbors where cooperative actions is necessary.

To fulfil these capacities, search and rescue robots require robust and adaptive fail-soft systems to achieve calculable reliability desired for operations under unstructured environment. In this respect Human immune system is useful for this purpose as it is a robust and adaptive decentralized system (Sompayrac, 1999); the function of its components and their interactions offer inspiring analogies for solving problems in different disciplines. The suppression mechanism between immune cells, for example, demonstrates the possibility of using simple local signals to generate useful global behaviors in a dynamically changing environment. This research exploits the mechanisms that give the biological immune system robustness and adaptabilities to develop a decentralized framework for controlling these search and rescue robots.

Based on the properties of modular robots, an immunity-based control framework is developed to coordination their activities. Modular robots are module-based systems that can be configured to accommodate different operation requirements (Marbach and Ijspeert, 2005; Groß, 2006; Kamimura, 2005). The ability to change shape dynamically during operation enables modular robots to navigate across larger gaps, creep through smaller voids and to climb over higher obstacles more effectively than conventional robots. The inherited decentralized nature of modular robots requires flexible decentralized control system that can adapt to its topological changes to take full advantages of modular robots' versatility. Immunity-based control systems inspired by the biological immune system exhibits essential characteristics for controlling such modular robots.
The decentralized nature plus the robustness and adaptability of immunity-based control system enable modular robots to operate effectively.

In this paper, the design of the General Suppression Control Framework (GSCF) that is inspired from the suppression hypothesis in discrimination theory of immunology is presented to control and coordinate heterogeneous modular robots. Computer simulations are conducted of using GSCF to mimic the basic operations of the immune system to control a cooperative group of search and rescue robots.

\section{HUMAN AND ARTIFICIAL IMMUNE SYSTEMS}

\subsection{Human Immune System}

Human immune system (Benjamini, 1996; Sharon, 1998) is a robust, efficient, and adaptive system that continuously acquires new knowledge of non-self cells, adjusts its responses against foreign antigens and suppresses destructive actions against self cells (Elgert, 1996). In summary, there are four main functions exhibited by the human immune system (Playfair and Chain, 2001):

(1) Clonal Selection Specific cells in the immune system produce antibodies that fit only one specific type of antigen. When these specific cells spotted the existence of a recognizable antigen, they proliferate to clone copies of themselves with identical characteristics.

(2) Immunological Memory Unique antibodies that successfully destroyed foreign invaders are maintained within the system to become part of the distributed immunological memory of the system. These antibodies can be activated quickly to protect the body when the same antigen revisit.

(3) Antibody Diversity Unique antibodies for specific antigens are produced by a modular design process which mixes and matches segments of cell genes. The result of this mix and match strategy is a small number of gene segments that can create incredible antibody diversity.

(4) Cell Discrimination Non-self cells from self cells 
are discriminated. In this context, self cells are the good cells that work inside our body and non-self cells (antigens) are external elements that does harm. The distinction and the recognition of foreign antigens are done by the B-Cells and T-Cells. B-cells are lymphocytes that mature in the bone marrow, and T-cells are white blood cells that mature in the thymus. These two kinds of cells allow the system to identify harmful molecules to kill and to leave the good molecules (self-cells) untouched. These powerful functions together form the basis of immune system; their underlying architectures offer inspiring analogies in many aspects for developing reliable decentralized systems.

\subsection{Artificial Immune System}

Artificial Immune Systems (AIS) (de Castro, 2001; De Castro and Timmis, 2003) is a new computational paradigm inspired from its biological counterpart. Researchers have applied AIS to solve a wide variety of problem. Lau \& Wong (2004) developed a control framework to improve the efficiency of a distributed material handling system. Neal et al. (2006) developed a model for integration of low-level responses to damage and component failure in robots, based on the notion of artificial inflammation and an extensible, sub-symbolic mechanism for modulating high-level behavior. Dasgupta et al. (2004) exploited negative selection algorithm to detect abnormalities in aircrafts. Cserey et al. (2004) developed an AIS real-time visual analysis system for surveillance based on the behavior of T-cells.

The development of computation paradigms based on AIS often exploit and mimic the four main functions of biological immune system by embedding various computational techniques and algorithms (Sipper, 2003). Many of these paradigms had successfully implemented to deal with decentralized systems to perform learning, data manipulation, abnormality detection, object classification and pattern matching operations.

In our study, we developed an AIS paradigm for the control and coordination of modular robots that has the ability to search for abnormalities under the general suppression control framework. We capitalize the decentralized nature of AIS together with its robustness and adaptability to achieve the characteristics of the modular robots. Though the theme of this research focuses in search and rescue domain, an ultimate objective is to develop a generic decentralized control framework for modular robots base on the AIS computation paradigm.

\section{General Suppression Control Framework}

The GSCF (Ko et al., 2005) is designed based on the analogy of the immunological suppression hypothesis in the discrimination theory (Aickelin, 2003). The major recognition and reaction functions of the acquired immunological response are performed by T-lymphocytes (T-cells) and B-lymphocytes (B-cells) which exhibit specificity towards antigen. B-cells synthesize and secrete into the bloodstream antibodies with specificity against the antigen, the process is termed Humoral Immunity. The T-cells do not make antibodies but seek out the invader to kill; they also help B-cells to make antibodies and activate macrophages to consume foreign matters. Acquired immunity facilitated by T-cells is called Cellular Immunity.

When a T-cell receptor binds to a peptide with high affinity presented by an APC (Antigen Presenting Cells), such as macrophages, the $\mathrm{T}$-cell recognized the antigen become mature and it has to decide whether to attack the antigen aggressively or to tolerate it in peace. An important decision factor is the local environment within which the T-cell resides. The present of inflammatory cytokine molecules such as interferon-gamma (INF- $\gamma$ ) (Sharon, 1998) in the environment tend to elicit aggressive behaviors of T-cells, whereas the anti-inflammatory cytokines like IL-4 and IL-10 tend to suppress such behavior by blocking the signaling of aggression. In brief, a T-cell matured after recognizing an antigen does not start killing unless the environment also contains encouraging factors for doing so. In addition, after a mature T-cell developed the behavior, it will emit humoral signals that have slower transmission speed but longer lasting effect than cellular signals to convert others to join.

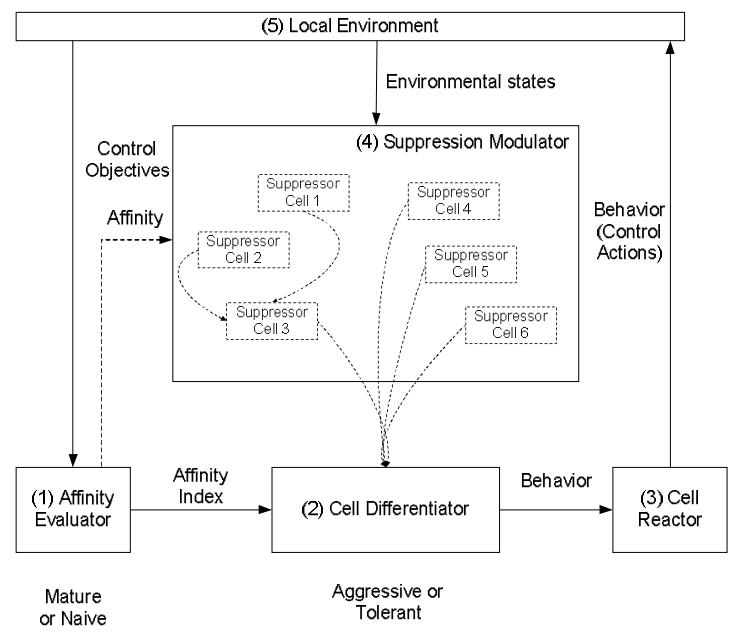

Fig.1 The General Suppression Control Framework (Dashed lines represent humoral signal, where solid lines represent cellular signals)

Our analogy infers each module of the modular robot is an autonomous $\mathrm{T}$-cell that continuously reacts to the changing environment and affects the functioning of other cells through the environment. In particular, T-cell's functions are divided into three separate components, the Affinity Evaluator, Cell Differentiator and the Cell Reactor. There are five main components in GSCF; they are Affinity Evaluator, Cell Differentiator, Cell Reactor, Suppression Modulator, and the Local Environment (Fig. 1).

(1) Affinity Evaluator evaluates information in the Local Environment against the objective and output an affinity index. The function of this component is similar to the immune discrimination function of biological immune systems, which helps to differentiate between 
self and non-self cells. Affinity Evaluator can affect the Cell Differentiator in two ways, the first is to directly send a cellular signal that indicates the affinity level, and the second is to send humoral signals to Suppressor Cells (SC) in the Suppression Modulator.

(2) Cell Differentiator- - evaluates inputs from the Affinity Evaluator and Suppression Modulator to decide the type of behavior to react. The decided behavior is sent to Cell Reactor using cellular signaling. The component can also send humoral signals directly to influence the Local Environment. The functioning of the Cell Differentiator is similar to the cell differentiation mechanism, in which cells develop aggressive or tolerant behavior in response to the type of cytokines present in the environment. When activated, these cells also release humoral signals to convert nearby cells to duplicate their behavior.

(3) Cell Reactor reacts to the cellular signal from the Cell Differentiator and execute the corresponding behaviors which take effect in the Local Environment. This component is the part that actually does the killing like activated aggressive T-cells.

(4) Suppression Modulator-consists of a collection of Suppressor Cells. The function specific Suppressor Cells continuously react to external stimulants to adjust their specific function, perform proliferation and bond and recombine with other Suppressor Cells to develop new specific functions. The function of Suppression Modulator is analogous to the cytokine signaling mechanism that performs intercellular communication, and to elicit or suppress aggressive behaviors in the T-cells. In this framework, the Suppression Modulator acquires information from the Local Environment and the Affinity Evaluator. This information is available to all Suppressor Cells within the modulator.

(5) Local Environment - is where interactions between different components take place. The importance of this component within the framework is to act as an interface that links to the Global Environment which contains other Local Environments with different sets of Suppression Modulators.

\section{EXPERIMENTATION}

Simulation study was performed to investigate the function of the GSCF in controlling and coordinating a number of distributed agents that represent the modular robots. The simulation consists of five distributive classes of agents working in close cooperation. Though the simulation constructed in MATLAB can be used to study a large number of governing variables, this study focuses on studying system performance against cell diversity.

The multi-agent system under simulation that builds on the immune mechanism has four classes of active agents and one passive agent in which the active agents are Explorer, Expert, Action Agent, and Backup Agent, and the passive Target Agent that represents the virus. Table 1 summarizes the biological counterparts of these elements.
In the simulation, the role of macrophages is represented by the Explorers, which is a class of agent which constantly explores the search space to look for the presence of Targets. When a target is found, an Explorer will stand by the target, examine it, and broadcast its information to nearby Experts. Similar to macrophages display pieces of virus to attract helper T-cells, Explorers can broadcast signals to all agents within a defined perimeter. Subsequently, an Expert with the specific affinity recognizes the broadcasted signal and move towards the Explorer. When an Expert is teamed with an Explorer, Target information is confirmed and the team will broadcast Global information to invite Action Agents to come to take action. At the same time Action Agents will pass on the global signal to other Action Agents to raise the local action power. This process is similar to the action of helper T-cells calling for killer T-cells to multiply. In biological systems, cells can proliferate to any number when needed, but in robotics, the number of agents in the field is often fixed, the increase of local action power can only be achieved by pulling agents from nearby regions.

While Action Agents are inviting more fellows to the region, it continues broadcasting global signals to Backup Agents. Once a Backup Agent receives the signal, it will start to label all other objects with identical features as described in the Target information, while passing the digital label to fellow Backup Agents. This process means Backup Agents can be activated in two ways, either by global Target information from Action Agents or by digital labels issued by fellow Backup Agents. This process is similar to the multiplication of B-cells, and its production of antibodies.

After the Targets are digitally labeled, they can be "legally" tackled. Action Agents then tackle all digitally labeled Targets within their proximity. Fig. 2 describes the control and interactions between the agents.

Based on the control logic, Fig. 3 illustrates the basic design of the implementation of the simulation based on GSCF. The five agents' behaviors are treated as interdependent suppressor cells inside of the suppression modulator. The affinity evaluator is used to monitor number of iterations the simulation must repeat before reporting the result. Cell differentiator collects behavioral status from the Action Agents in the suppressor cell and forward to the cell reactor for display. The local environment continues to be a place for system interactions.

Tab.1 Immune cell classes represented by simulated agents

\begin{tabular}{c|c}
\hline Natural Immune System & Search and Rescue System \\
\hline Pathogens & Targets \\
\hline Macrophages & Explorers \\
\hline Helper T-cells & Experts \\
\hline Killer T-cells & Action Agents \\
\hline B-cells & Backup Agents \\
\hline
\end{tabular}

\section{SIMULATION RESULTS AND DISCUSSIONS}

The simulations study the factors controlling the overall 
system performance with the focus to reveal the overall system performance with respect to the changes in the number of agents in different agent classes. Experiments consider the five agent classes, namely, Explorer, Expert, Action Agent, Backup Agent and Target classes. Except for the agent class under consideration that the number of agent deployed is varied, all other agent classes are set to have twenty agents each. To add reliability to the experiment results, the agent class needs to repeat the same set of experiment ten times.

In this simulation, system performance is measured by number of iterations required to tackle all the Targets. Test results on system performance against the two key elements, namely, the Action Agents and Explorers are shown in Fig. 4 and 5. The graph at the upper left hand corner of Fig. 4 is a scatter plot of all raw data collected. The $\mathrm{x}$-axis is the number of Action Agents in the system, where $y$-axis is number of iterations required to tackle all Targets. As the simulation runs from $1-20$ Action Agents, there are twenty discrete columns of scatter points on the graph. Each column in the graph has ten points, denoting there were ten repeated trials with the same number of Action Agents.

Tab. 2 list the number of iterations required to tackle all Targets with one Action Agent at ten different trials. Notice the number of iterations ranges from a minimum of 1457 (2nd trial) to a maximum of 8298 (4th trial). To obtain a more representative result, the highest and lowest results are removed to produce the graph on the upper right hand corner labeled "High-low Cut-off".

The graphs (Fig. 4) show the average iterations required to tackle all Targets, the decrease in average iterations taken as the number of Action Agents increase fits an exponential curve. The "High-low Cut-off Average" graph shows that when the number of Action Agents increases to about five, its effect on improving system performance starts to flatten out.

For Explorer agent class, Fig. 5 shows that the exponential curve (middle-right) quickly loses its affect on overall system performance as soon as number of Explorer agents increase to two. In fact, the "High-low Cut-off Semi-log graph suggest that population changes in Explorers plays a minimal role to the overall system performance. One possible explanation to this phenomenon is that Explorers are the first class of agents in the whole search and rescue process; therefore it is not affected by the performance of other agents. The reason why the number of iterations drops significantly after the first trial is because even when there are only two Explorers in the environment, the chances for them to find one of the twenty Targets are basically the same as when there are twenty Explorers finding one of the twenty Targets, and whenever an Explorer finds a Target, the possibility of having an Expert nearby is high (twenty Expert in the environment), therefore Explorers will not become a bottle neck to the exploration process even when initial number of agents in its class is low.

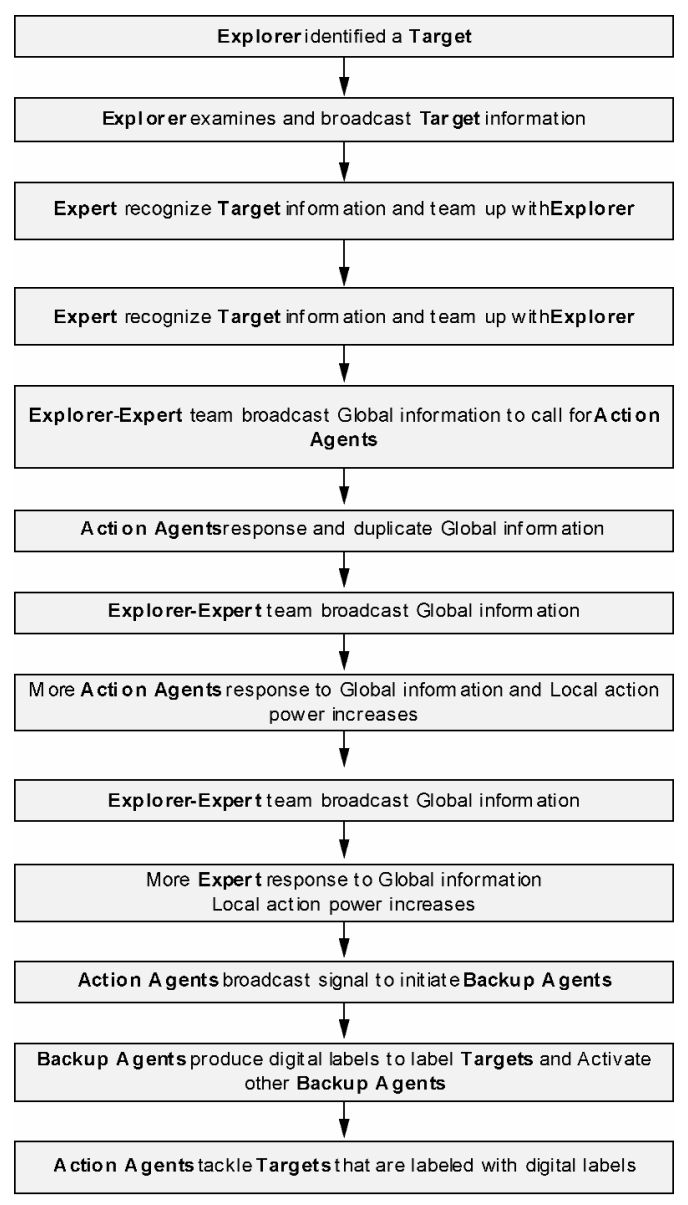

Fig.2 Action sequence of the search and rescue robot team

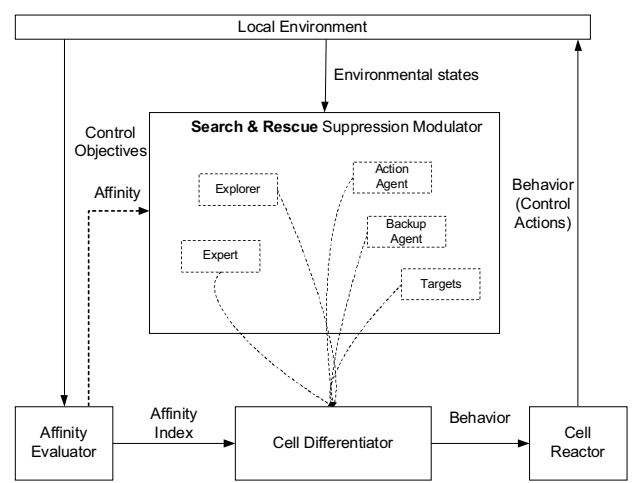

Fig.3 The architecture of the search and rescue robot control framework

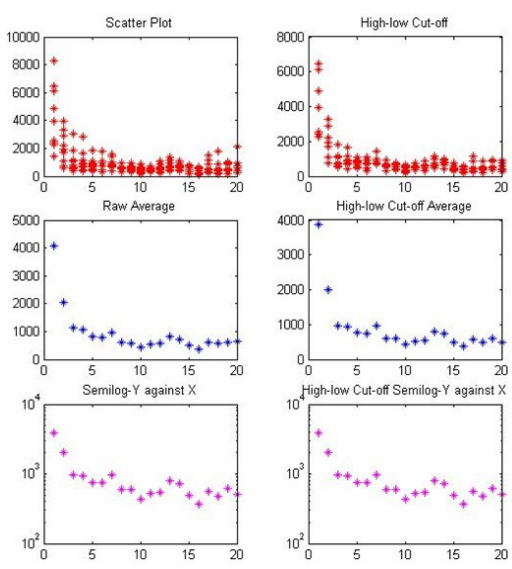

Fig.4 Number of iterations required to tackle all Targets with respect to the number of Action Agents 

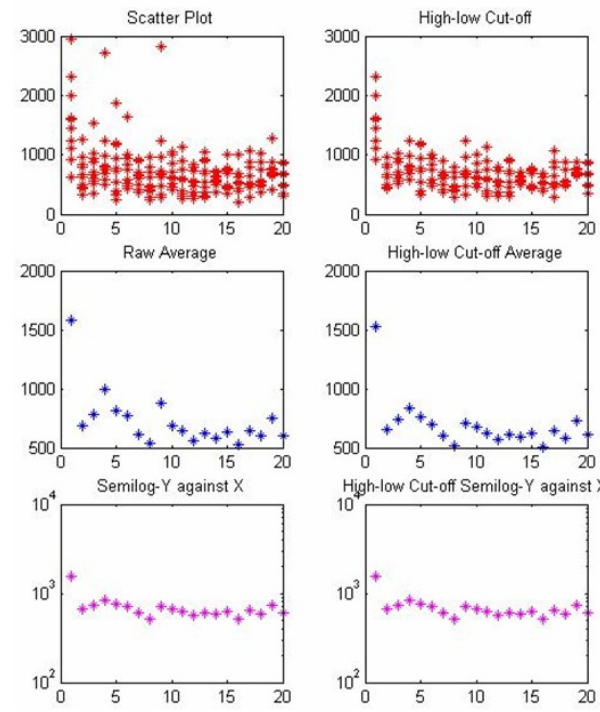

Fig.5 Number of iterations required to tackle all Targets with respect to the number of Explorers

\section{CONCLUSION}

This paper has presented a framework for control and coordination of search and rescue robots that is capable of modeling large scale heterogeneous systems based on the computation paradigm of the biological immune system. A simulation based on GSCF was implemented to investigate the impact of agent diversity on overall system performance. The behaviors of agents representing the modular robots were designed in respect to the cell behaviors of the immune system. It is found that GSCF is able to impose a self-regulated mechanism for the cooperating agents in tackling tasks. The simulation results also demonstrate that through the sharing of salient information, efficient cooperation between agents can be achieved.

We are currently investigating the effect of factors such as group diversity and social entropy on the overall performance of the system. Developing a quantitative method to evaluate diversity would help the investigation on diversity's impact on performance and conversely, the impact of system functions on diversity. With the results obtained so far, it his hoped that the development of a fully decentralized control model based on the AIS paradigm can be achieved.

Tab.2 Number of iterations taken to tackle all Targets with one Action Agent in 10 different trials

\begin{tabular}{c|c}
\hline i-th iteration & Number of Iterations \\
\hline $1^{\text {st }}$ & 2293 \\
\hline $2^{\text {nd }}$ & 1457 \\
\hline $3^{\text {rd }}$ & 3955 \\
\hline $4^{\text {th }}$ & 8298 \\
\hline $5^{\text {th }}$ & 4889 \\
\hline $6^{\text {th }}$ & 2430 \\
\hline $7^{\text {th }}$ & 2568 \\
\hline $8^{\text {th }}$ & 6125 \\
\hline $9^{\text {th }}$ & 2263 \\
\hline $10^{\text {th }}$ & 6461 \\
\hline
\end{tabular}

\section{REFERENCES}

[1] Aickelin U, Bentley P, Cayzer S. et al. Danger theory: the link between AIS and IDS: Proceeding of 2nd International Conference on Artificial Immune Systems, (ICARIS 2003).

[2] Benjamini, E., G. Sunshine, S. Leskowitz. Immunology: A Short Course[M], New York, Wiley-Liss, USA.

[3] Cserey G, Porod W, Roska T. An artificial immune system based visual analysis model and its real-time terrain surveillance application: Proc. of 3rd International Conference on Artificial Immune Systems (ICARIS 2004), Catania, Italy.

[4] de Castro L N. An Introduction to the Artificial Immune Systems, from Catholic University of Santos [EB/OL] [2006-6-2]http://www.dca.fee.unicamp.br/ lnunes/AIS.html

[5] de Castro L N, Timmis J. Artificial immune Systems as a Novel Soft Computing Paradigm[J]. Softing Computing, 2003, 7(8): 526-544.

[6] Elgert K D. Immunology: Understanding the Immune System, New York: Wiley-Liss.

[7] Groß R, Tuci E. Object transport by modular robots that self-assemble: Proceedings of the 2006 IEEE International Conference on Robotics and Automation, Orlando, Florida, May, 2006.

[8] Kamimura A, Kurokawa H, Yoshida E, et al. Automatic locomotion design and experiments for a Modular robotic system[J]. Mechatronics, IEEE/ASME Transactions on: 314325.

[9] KrishnaKumar D S, Wong K D, Berry M. Negative Selection algorithm for aircraft fault detection: Proc. of 3rd International Conference on Artificial Immune Systems (ICARIS 2004). Catania, Italy, 13 - 16 September. pp. 1-13.

[10] Ko A, Lau H Y K, Lau T L. An immuno control framework for decentralized mechatronic control[J]. International Journal of Unconventional Computing, Special Issues, 2005: 225-280.

[11] Lau H Y K, V W K Wong. Immunologic responses manipulation of AIS agents handling: Proc. of 3rd International Conference on Artificial Immune Systems (ICARIS 2004), Catania, Italy, 13 - 16 September: 65-7.

[12] Marbach D, Ijspeert A J. Online optimization of modular robot locomotion: Proceedings of the IEEE International Conference on Mechatronics \& Automation, Niagara Falls, Canada.

[13] Neal M, Feyereisl J, Rascun`a R. et al. Don't touch me, I'm fine: robot autonomy using an artificial innate immune system: Proc. of 5th International Conference on Artificial Immune Systems (ICARIS 2006)].

[14] Playfair J H L, Chain B M. Immunology at a Glance[M]. Bodmin, Cornwall: Blackwell Science.

[15] Sharon, J. Basic Immunology[M] Pennsylvania, USA: Williams \& Wilkins, 1998.

[16] Sipper M. Machine Nature[M]//the Coming Age of Bio-inspired Computing, New York: McGraw-Hill.

[17] Sompayrac L M. How the Immune System Works[M], Massachusetts USA: Blackwell Publishing. 